\title{
PAl-1 promotes the accumulation of exudate macrophages and worsens pulmonary fibrosis following type II alveolar epithelial cell injury
}

\author{
John J Osterholzer,',2 Paul J Christensen,',2 Vibha Lama,' Jeffrey C Horowitz,' Noboru Hattori,,3 \\ Natalya Subbotina,' Andrew Cunningham,' Yujing Lin,' Benjamin J Murdock,',2 Roger E Morey,2 \\ Michal A Olszewski, ${ }^{\prime, 2}$ Daniel A Lawrence, ${ }^{4}$ Richard H Simon' and Thomas H Sisson'* \\ I Division of Pulmonary and Critical Care Medicine, Department of Internal Medicine, University of Michigan Medical School, Ann Arbor, MI, USA \\ 2 Research Service, Ann Arbor VA Health System, Department of Veterans Affairs Health System, Ann Arbor, MI, USA \\ 3 Department of Molecular and Internal Medicine, Graduate School of Biomedical Sciences, Hiroshima University, Japan \\ ${ }^{4}$ Division of Cardiology, Department of Internal Medicine, University of Michigan Medical School, Ann Arbor, MI, USA
}

* Correspondence to: Thomas H Sisson, University of Michigan Medical Center, Department of Internal Medicine, Division of Pulmonary and Critical Care Medicine, I 150 West Medical Center Drive, 630I MSRB III, Ann Arbor, MI 48 I09-5642, USA. e-mail: tsisson@umich.edu

\begin{abstract}
Fibrotic disorders of the lung are associated with perturbations in the plasminogen activation system. Specifically, plasminogen activator inhibitor-1 (PAl-1) expression is increased relative to the plasminogen activators. A direct role for this imbalance in modulating the severity of lung scarring following injury has been substantiated in the bleomycin model of pulmonary fibrosis. However, it remains unclear whether derangements in the plasminogen activation system contribute more generally to the pathogenesis of lung fibrosis beyond bleomycin injury. To answer this question, we employed an alternative model of lung scarring, in which type II alveolar epithelial cells (AECs) are specifically injured by administering diphtheria toxin (DT) to mice genetically engineered to express the human DT receptor (DTR) off the surfactant protein C promoter. This targeted AEC injury results in the diffuse accumulation of interstitial collagen. In the present study, we found that this targeted type II cell insult also increases PAI-1 expression in the alveolar compartment. We identified AECs and lung macrophages to be sources of PAl-1 production. To determine whether this elevated PAI-1 concentration was directly related to the severity of fibrosis, $\mathrm{DTR}^{+}$mice were crossed into a PAI-1-deficient background (DTR ${ }^{+}:$PAI-1 ${ }^{-/-}$). DT administration to $\mathrm{DTR}^{+}$: PAI-1-/- animals caused significantly less fibrosis than was measured in $\mathrm{DTR}^{+}$mice with intact PAl-1 production. PAI-1 deficiency also abrogated the accumulation of CD11 $\mathrm{b}^{+}$exudate macrophages that were found to express PAI- 1 and type- 1 collagen. These observations substantiate the critical function of PAl-1 in pulmonary fibrosis pathogenesis and provide new insight into a potential mechanism by which this pro-fibrotic molecule influences collagen accumulation.
\end{abstract}

Copyright (c) 2012 Pathological Society of Great Britain and Ireland. Published by John Wiley \& Sons, Ltd.

Keywords: PAl-1; lung; fibrosis; macrophage

Received 26 October 201 I; Revised I2 December 20II; Accepted I2 January 2012

No conflicts of interest were declared.

\section{Introduction}

Both acute and chronic fibrotic disorders of the lung, including acute lung injury/acute respiratory distress syndrome (ALI/ARDS) and idiopathic pulmonary fibrosis (IPF), are associated with significant morbidity and mortality. Few treatments have been shown to definitively modulate the severe course of these diseases [1-4]. In ARDS, the mortality remains at 25-30\% despite improvements in mechanical ventilation, and $60-70 \%$ of individuals afflicted with IPF will die within 5 years of their diagnosis [5-12]. Therefore, it is imperative that we better understand the pathogenic mechanisms underlying fibrosis of the lung in order to define new treatment strategies.
Perturbations of the plasminogen activator pathway are common in conditions associated with pulmonary fibrosis. Specifically, in both ARDS and IPF, plasminogen activation is inhibited secondary to an increased expression of plasminogen activator inhibitor-1 (PAI-1) relative to the plasminogen activators [13-17]. Furthermore, elevated levels of PAI-1 in patients with ALI are associated with increased mortality [18]. Animal studies using bleomycin-induced lung injury reveal very similar derangements in plasminogen activation [19-21]. The bleomycin model has also been used to establish a causal link between the impairment in plasminogen activation and the severity of lung collagen accumulation. In particular, restoration of plasminogen activator activity [whether it is achieved by: (a) the 
exogenous administration of uPA; (b) increased intrapulmonary uPA expression; or (c) PAI-1 deficiency] significantly decreases bleomycin-induced lung collagen accumulation [22-27]. On the other hand, further suppression of plasminogen activation through the constitutive over-expression of PAI-1 significantly worsens lung fibrosis following bleomycin injury [22].

With the exception of a single recent report, the casual relationship between the plasminogen activation system and the extent of fibrosis that follows lung injury has been entirely established in the bleomycin animal model [28]. To address this shortcoming, we tested the role of PAI- 1 in the development of pulmonary fibrosis, using a model that specifically injures type II alveolar epithelial cells [29]. This model was motivated by the prevalent type II cell abnormalities seen in histopathological sections of IPF patients and the link between type II alveolar cell-specific gene mutations and familial pulmonary fibrosis [30-34]. In this model, mice genetically engineered to express the human diphtheria toxin receptor (DTR) on their type II alveolar epithelium are given serial administrations of diphtheria toxin (DT). In response to the DT treatment, the DTR-expressing transgenic mice develop significant fibrosis [29].

Here we report that a targeted insult of the type II alveolar epithelium induces a marked increase in intrapulmonary PAI-1 levels. Furthermore, we demonstrate that PAI-1 deficiency significantly attenuates the collagen accumulation and mortality that results from this specific injury. Finally, we provide evidence for a novel mechanism, the accrual of exudate macrophages in the lung, by which PAI-1 promotes fibrogenesis. These observations further substantiate the role of the plasminogen activator system in pulmonary fibrosis and suggest that derangements in this system could represent a common pathogenic pathway shared by the many different causes of lung scarring.

\section{Materials and methods}

\section{Animals}

All animal experiments were performed in accordance with institutional guidelines set forth by the University Committee on the Use and Care of Animals (UCUCA). Mice deficient in PAI-1 (PAI-1 ${ }^{-/-}$) were supplied by Dr Peter Carmeliet (University of Leuven, Belgium) and had been backcrossed with $\mathrm{C} 57 \mathrm{BL} / 6$ mice for at least eight generations [35]. Transgenic mice expressing the human diphtheria toxin receptor (DTR) from a murine SPC promoter were generated on a C57BL/6 background. These mice were repeatedly crossed with PAI-1 ${ }^{-/-}$mice to generate offspring with a single copy of the DTR gene and that were PAI-1-deficient $\left(\mathrm{DTR}^{+}\right.$: PAI-1-/-). Control C57BL/6 mice [wild-type (WT)] were from Jackson Laboratories (Bar Harbor, ME, USA).

\section{Assessment of mouse genotypes}

The presence of the DTR construct and the targeted gene deletion of PAI-1 were detected using PCR, as previously described [25,29].

\section{Diphtheria toxin administration}

Six to eight week-old $\mathrm{DTR}^{+}$: $\mathrm{PAI}-1^{+/+}, \mathrm{DTR}^{+}$: PAI$1^{-/-}, \mathrm{DTR}^{-}:$PAI-1 ${ }^{+/+}(\mathrm{WT})$ and $\mathrm{DTR}^{-}:$PAI-1 ${ }^{-/-}$ (PAI-1 knock-out) mice were injected i.p. with DT once daily for 14 days at doses of 10.0 and $12.5 \mu \mathrm{g} / \mathrm{kg}$. Control mice were injected for the same duration with $100 \mu \mathrm{l}$ PBS alone.

\section{Bronchoalveolar lavage}

BAL fluid was generated by instilling $1.0 \mathrm{ml}$ sterile PBS via a blunted 18-gauge needle into the trachea. Recovery of the fluid was consistently $70-80 \%$ of the total. The BAL fluid was then centrifuged at $4000 \times g$ for $10 \mathrm{~min}$, the supernatant was removed and the samples were stored immediately at $-80^{\circ} \mathrm{C}$.

\section{BAL fluid PAI-1 concentration measurements}

Endogenous murine PAI-1 concentrations were measured in BAL fluid using a carboxylated microspherebased ELISA, as previously described [29].

\section{Hydroxyproline assay}

Hydroxyproline content of the lung was measured as previously described [25].

\section{Lung histology}

The left lung was inflation-fixed at $25 \mathrm{~cm} \mathrm{H} \mathrm{H}_{2} \mathrm{O}$ pressure with $10 \%$ neutral-buffered formalin, removed en bloc, further fixed in $10 \%$ neutral-buffered formalin overnight and then paraffin-embedded. Sections $(5 \mu \mathrm{m})$ were stained using Masson's trichrome, picrosirius red and immunohistochemistry methods.

\section{Type II alveolar epithelial cell PAI-1 expression}

We measured the expression of PAI-1 by type II alveolar epithelial cells following injury using two different approaches: in both methods, type II AECs were isolated as previously described [36]. In the first assay, uninjured cells were isolated from $\mathrm{DTR}^{+}$and $\mathrm{DTR}^{-}$mice; $5.0 \times 10^{5}$ cells were plated in a 24well tissue culture plate. After $48 \mathrm{~h}$, a subset of cells from each genotype was exposed to DT at a dose of $1.0 \mu \mathrm{g} / \mathrm{ml}$ for $24 \mathrm{~h}$. RNA was harvested from the cells and PAI- 1 expression was assessed via $\mathrm{qRT}-\mathrm{PCR}$ and normalized to GAPDH. PAI-1 message levels from the treated cells were compared to untreated cells of the same genotype. In the second assay, $\mathrm{DTR}^{+}$and $\mathrm{DTR}^{-}$ mice were treated with DT at $10 \mu \mathrm{g} / \mathrm{ml}$ for 14 days. A control group of $\mathrm{DTR}^{-}$mice was treated with PBS for 14 days. Type II AECs were isolated from each group and $2.0 \times 10^{6}$ cells were cultured. After $48 \mathrm{~h}$, RNA 
was harvested from the cells and PAI-1 expression was assessed via qRT-PCR and normalized to GAPDH. PAI-1 message levels from the DT-treated groups were compared to the PAI-1 expression of cells from the control mice.

\section{Immunofluorescence for PAI-1 and SPC}

Sequential co-staining was performed on sections for surfactant protein C (SPC), followed by PAI-1. Briefly, paraffin-embedded slides underwent antigen retrieval with an antigen-unmasking solution. The slides were then stained, using Universal ABC Vectastain Kit according to the manufacturer's instructions, and tyramide fluorescence peroxidase substrates (tetramethylrhodamine $=$ red and fluorescein tyramide $=$ green) were used to achieve the final stain intensity. DAPI nuclear stain was performed prior to mounting.

\section{Tissue collection for lung leukocyte quantification}

The lungs were perfused via the right heart, using PBS containing $0.5 \mathrm{mM}$ EDTA until the pulmonary vessels were grossly clear. The lungs were then excised, minced and enzymatically digested to obtain a single cell suspension of lung leukocytes, as previously described [37].

\section{Antibody staining and flow cytometric analysis}

Staining, including blockade of Fc receptors, and analysis by flow cytometry was performed as described previously [37]. Specific gating strategies identifying lung macrophage subpopulations (autofluorescent $\mathrm{CD}_{11 \mathrm{c}^{+}}$: $\mathrm{CD}_{11 \mathrm{~b}^{-}}$alveolar macrophages and autofluorescent $\mathrm{CD}_{11} \mathrm{c}^{+}$: $\mathrm{CD}_{11 \mathrm{~b}^{+}}$exudate macrophages and monocyte subsets) and CD11 ${ }^{-}: \mathrm{CD}_{11 \mathrm{~b}^{+}} \mathrm{F} 4 / 80^{+} \mathrm{Ly}-$ $6 C^{\text {high }}$ monocytes [38]. Intracellular staining for type-1 collagen was performed as previously reported [38,39].

\section{Macrophage isolation}

Total lung leukocytes were obtained (as above) from the lungs of individual DT-treated $\mathrm{DTR}^{-}$mice $(n=3)$ or $\mathrm{DTR}^{+}$mice $(n=3)$ mice following 14 days of DT treatment. Lung leukocytes from each strain of mice were then pooled and subjected to fluorescenceactivated cell sorting to isolate specific macrophage subsets to $>95 \%$ purity, using the same antibody staining and gating strategies previously described [38,39].

\section{RT - PCR}

Total macrophage RNA was prepared and specific gene expression assessed using a previously described protocol [38].

\section{Statistical analysis}

The data are presented as mean \pm standard error of the mean (SEM). Statistics were performed using GraphPad Prism software. Mean values from the different groups were compared using a one-way analysis of variance with post hoc pairwise comparisons using the Newman-Kuels Multiple Comparison Test. $p<0.05$ was considered statistically significant.

\section{Results}

\section{BAL fluid PAI-1 concentration in DT-treated DTR-expressing mice}

To determine whether targeted type II cell injury induces PAI-1 expression, DT was injected for 14 days into either WT control mice $\left(\mathrm{DTR}^{-}\right)$or $\mathrm{DTR}-$ expressing mice $\left(\mathrm{DTR}^{+}\right)$. WT mice injected with i.p. PBS served as an additional control. Following the last injection on day 14, BAL fluid was collected from each animal and the concentration of PAI-1 was analysed. As demonstrated in Figure 1, DT treatment caused a significant three- to four-fold increase in PAI-1 levels in the $\mathrm{DTR}^{+}$mice compared to the groups of control animals. When mice were maintained for an additional 7 days after the last DT injection (day 21), the mean BAL fluid PAI-1 concentration in $\mathrm{DTR}^{+}$mice remained elevated but was no longer statistically different from the control groups.

\section{Lung collagen content}

After determining that DT-mediated type II alveolar epithelial cell injury induced intrapulmonary PAI-1 production in DTR-expressing mice, we next investigated whether PAI-1 deficiency would protect against the resultant lung collagen accumulation that occurs in this model. To address this question, we administered DT for 14 days at a dose of $12.5 \mu \mathrm{g} / \mathrm{kg}$ to $\mathrm{DTR}^{+}$mice that were transgenically deficient in PAI1 DTR $^{+}$: PAI- $\left.{ }^{-/-}\right)$. The extent of lung fibrosis in these animals following DT treatment was then compared to DT-treated DTR-expressing mice that were WT for PAI-1 $\left(\mathrm{DTR}^{+}\right.$: PAI- $\left.1^{+/+}\right)$. Control groups of mice included DT-treated $\mathrm{DTR}^{-}$mice that were either WT or deficient in PAI-1 expression $\left(\mathrm{DTR}^{-}\right.$: $\mathrm{PAI}-1^{+/+}$ and $\mathrm{DTR}^{-}$: PAI-1 ${ }^{-/-}$). We also included a group of $\mathrm{DTR}^{-}$: PAI- ${ }^{+/+}$mice that was treated for 14 days with i.p. PBS to establish baseline lung collagen levels. To quantify the extent of fibrosis, we harvested lungs and measured lung hydroxyproline on day 21 ( 7 days after the last DT treatment). We chose day 21 based on our prior report that demonstrated a 1.5-2-fold increase in lung collagen content in DT-treated $\mathrm{DTR}^{+}$mice at this time point. Consistent with these previously published data, we found that targeted type II cell injury caused a significant increase in lung hydroxyproline content in the $\mathrm{DTR}^{+}$: PAI- $1^{+/+}$mice (Figure 2 ). Also consistent with our previous results, mice that do not express DTR and are WT for PAI-1 had no change in their lung collagen content following DT injection. Finally, we found that PAI-1 deficiency significantly protected DTR-expressing mice from DT-induced lung 
A
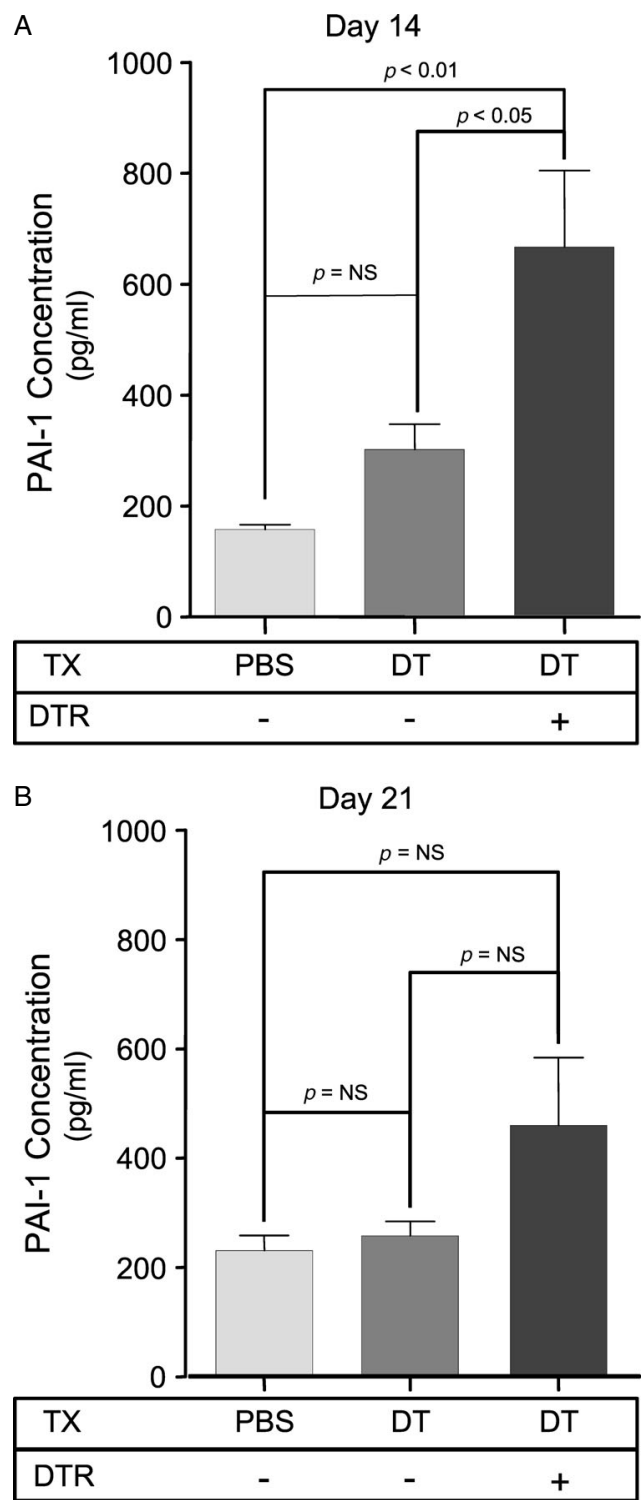

Figure 1. PAI-1 protein concentrations increase in mice with DTmediated alveolar injury. Diphtheria toxin was administered daily $(12.5 \mu \mathrm{g} / \mathrm{kg}$ in $100 \mu \mathrm{l}$ PBS by the i.p. route) for 14 days to either $\mathrm{DTR}^{-}$or DTR ${ }^{+}$mice. An additional control group of $\mathrm{DTR}^{-}$mice were injected with $100 \mu \mathrm{I}$ PBS for 14 days. BAL fluid was collected from each animal after the last DT injection on day $14(\mathrm{~A})$ and 1 week after the last DT injection on day 21 (B) and assayed by ELISA for PAl-1 protein concentration. Results are reported as mean \pm SEM $(n=6)$. Groups are compared with a one-way ANOVA and a Newman-Kuels post hoc multiple comparison test.

fibrosis. Importantly, in parallel studies, we also determined that PAI-1 deficiency significantly limited day 21 and day 28 lung collagen accumulation in $\mathrm{DTR}^{+}$ animals following 14 days of treatment with lowerdose DT(10.0 $\mu \mathrm{g} / \mathrm{kg})$ (data not shown).

\section{Survival}

We previously reported a $25 \%$ mortality rate over 21 days in DT-treated $\mathrm{DTR}^{+}$mice that received 14 days of treatment with the $12.5 \mu \mathrm{g} / \mathrm{kg}$ dose. In the current study, the $12.5 \mu \mathrm{g} / \mathrm{kg}$ dose again induced a $25 \%$ mortality in the $\mathrm{DTR}^{+}:$PAI- $1^{+/+}$animals. Another $35 \%$ of this group demonstrated significant weight

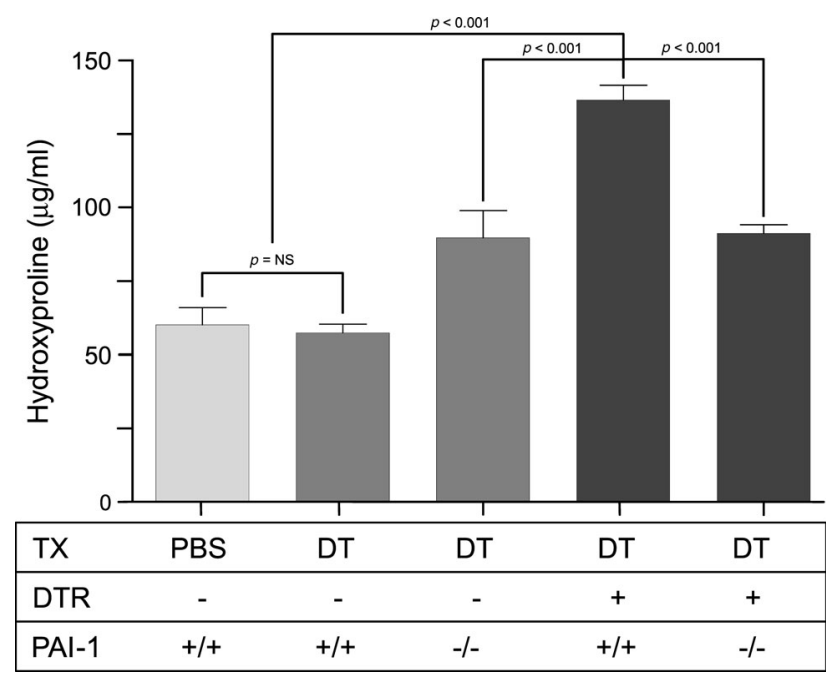

Figure 2. PAl-1 promotes lung collagen deposition following type II AEC injury. Diphtheria toxin $(12.5 \mu \mathrm{g} / \mathrm{kg})$ was administered for 14 days/protocol to four strains of mice: $1, \mathrm{DTR}^{-}: \mathrm{PAl}-1^{+/+} ; 2$, DTR $^{-}:$PAl- $1^{-/-} ; 3, \mathrm{DTR}^{+}: \mathrm{PAl}^{-1} 1^{+/+}$; and $\mathrm{DTR}^{+}: \mathrm{PAl}^{-1} 1^{-/-}$. An additional group of control $\mathrm{DTR}^{-}$: $\mathrm{PAl}-1^{+/+}$received daily i.p. injections of PBS for 14 days. Lungs were harvested on day 21 and analysed for hydroxyproline content. Results are reported as mean concentration (in $\mu \mathrm{g} / \mathrm{ml}) \pm \mathrm{SEM}(n=6-8)$. Groups are compared with a one-way ANOVA and a Newman-Kuels post hoc multiple comparison test.

loss $(>5.0 \mathrm{~g})$ at the end of the 21 day experimental period. These animals appeared pre-morbid and would have required euthanasia if the experimental period had continued for longer than 3 weeks (see Supporting information, Supplementary materials and methods, and Figure S1). In contrast, the DTR ${ }^{+}$: PAI- $1^{-/-}$group experienced no mortality at day 21 and the largest weight loss of any single animal in this group was only $1.4 \mathrm{~g}$.

\section{Lung histology}

To complement the quantitative hydroxyproline data on which statistical analyses were performed, we compared day 21 lung histology sections (two representative samples from each group) from the following three groups of mice following 14 days of DT treatment $\left(10 \mu \mathrm{g} / \mathrm{kg}\right.$ dose): (a) control mice (DTR ${ }^{-}$: PAI- $1^{+/+}$); (b) DTR ${ }^{+}$: PAI- $1^{+/+}$mice; and (c) DTR ${ }^{+}$: PAI-1 ${ }^{-/-}$mice. Sections were stained with both Masson's trichrome (Figure 3A) and picrosirius red (Figure 3B) techniques. As expected, lung sections from DT-treated control mice $\left(\mathrm{DTR}^{-}\right.$) revealed no evidence of lung injury or fibrosis (Figure $3 \mathrm{~A}$, top panels). In contrast, DT-treated $\mathrm{DTR}^{+}$: PAI- ${ }^{+/+}$mice demonstrate diffuse alveolar wall thickening with prominent blue staining and an accumulation of large cells with macrophage morphology (Figure 3A, middle panels). In contrast to the histological appearance of the $\mathrm{DTR}^{+}$: PAI- $1^{+/+}$lung sections, the DTR-expressing mice that were PAI-1-deficient $\left(\mathrm{DTR}^{+}\right.$: PAI-1 $\left.{ }^{-/-}\right)$had no appreciable fibrotic lesions (Figure 3A, bottom panels) and were indistinguishable from the control group. These 

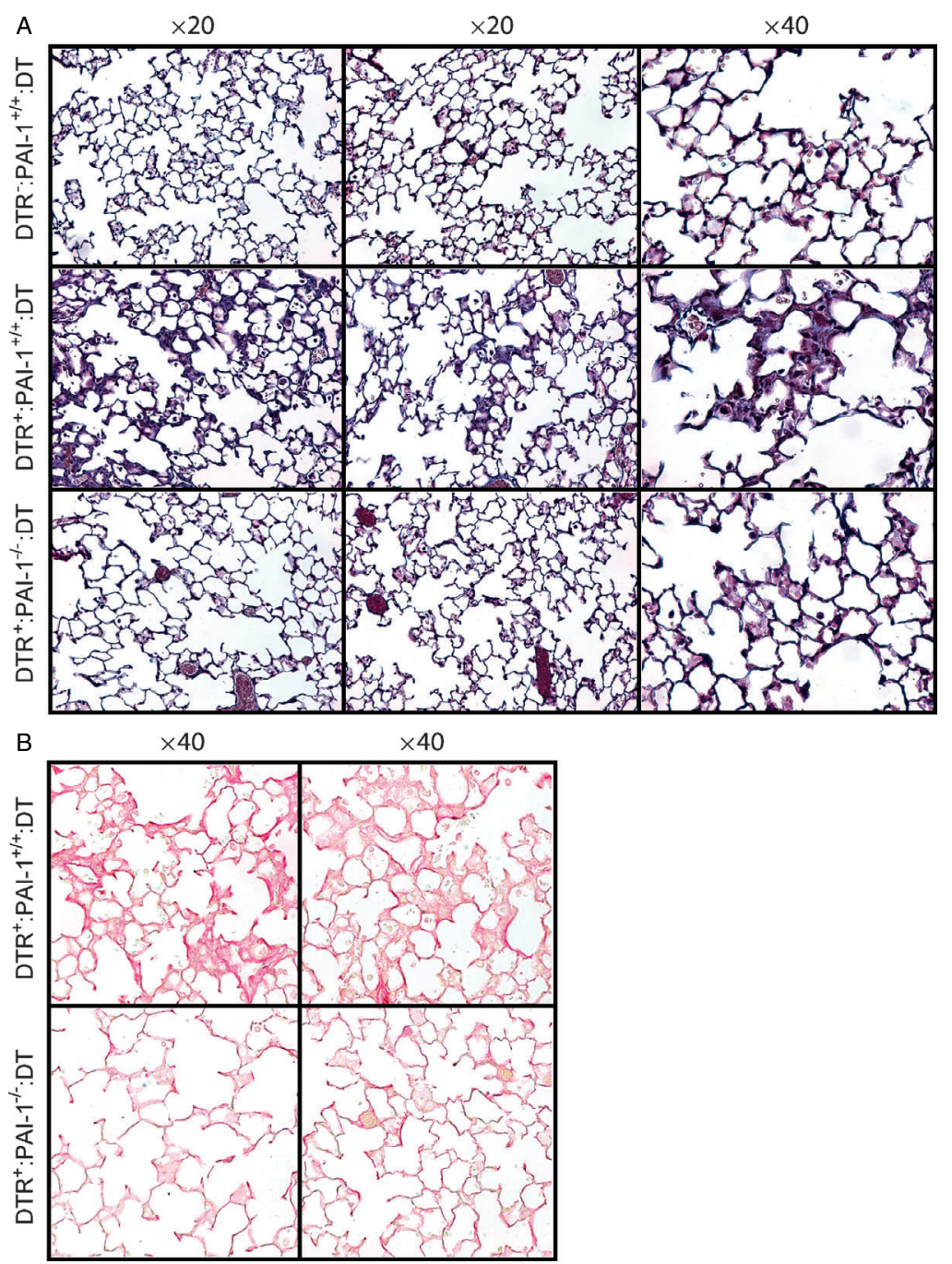

Figure 3. PAI-1 is associated with histopathological changes of pulmonary fibrosis following type II AEC injury. Diphtheria toxin $(10.0 \mu \mathrm{g} / \mathrm{kg})$ was administered for 14 days $/$ protocol to three strains of mice: 1 , DTR ${ }^{-}:$PAl-1+1+; 2 , DTR ${ }^{+}:$PAl- $1^{+/+} ;$and 3, DTR $^{+}$: PAI- $1^{-1-}$. On day 21, lungs were harvested and $5 \mu \mathrm{m}$ sections were generated for (A) Masson trichrome staining and (B) picrosirius red staining (DTR ${ }^{+}: \mathrm{PAl}-1^{+/+}$and $\mathrm{DTR}^{+}: \mathrm{PAl}-1^{-/-}$groups only). Representative images are shown for two mice in each group, taken with a $\times 20$ objective (Masson trichrome staining) and $\mathrm{a} \times 40$ objective (picrosirius red staining).

differences were further appreciated with collagenspecific picrosirius red staining (Figure 3B).

\section{Immunostaining for PAI-1}

Our observation that PAI-1 levels were increased in the lavage fluid of DT-treated $\mathrm{DTR}^{+}$mice led us to hypothesize that the injured type II alveolar epithelium may be a source of the PAI-1 production. We thus performed immunofluorescence with antibodies to PAI-1 and SPC on day 21 lung sections from three groups of DT-treated mice: (a) DTR ${ }^{-}$: PAI- $1^{+/+}$mice; (b) $\mathrm{DTR}^{+}$: PAI- $1^{+/+}$mice; and (c) $\mathrm{DTR}^{+}: \mathrm{PAI}-1^{-/-}$ mice (Figure 4). Images were then merged in order to assess for cells that stained positively for both markers. In the lung sections obtained from DT-treated $\mathrm{DTR}^{-}$: PAI- $1^{+/+}$control mice (Figure 4, top panels), a modest amount of PAI-1 staining was appreciable in SPC-expressing cells. In the lung sections obtained from DT-treated DTR ${ }^{+}$: PAI- $1^{+/+}$mice, we found PAI1 staining to be increased throughout the interstitium of the lung and within specific cells (Figure 4, middle panels). Some of these PAI-1-expressing cells also stained positively for SPC, whereas other cells were negative for SPC. Lung sections taken from DT-treated $\mathrm{DTR}^{+}$: PAI-1 ${ }^{-/-}$mice displayed minimal immunofluorescence for PAI-1 as expected (Figure 4, bottom panels). On the other hand, more prominent SPC staining was observed in the $\mathrm{DTR}^{+}$: PAI-1 ${ }^{-/-}$group compared to the DT-treated $\mathrm{DTR}^{+} / \mathrm{PAI}-1^{+/+}$mice, suggesting that PAI-1 deficiency may enhance the recovery of type II cells following DT treatment.

\section{PAI-1 expression by injured type II alveolar epithelial cells}

To verify that DT-mediated injury enhances PAI-1 expression by type II AECs, we employed two separate experimental approaches. First, we isolated type II AECs from $\mathrm{DTR}^{+}$and $\mathrm{DTR}^{-}$mice and cultured them in vitro. After $48 \mathrm{~h}$, we exposed subsets of the transgenic and WT cells to DT for $24 \mathrm{~h}$. The cells 


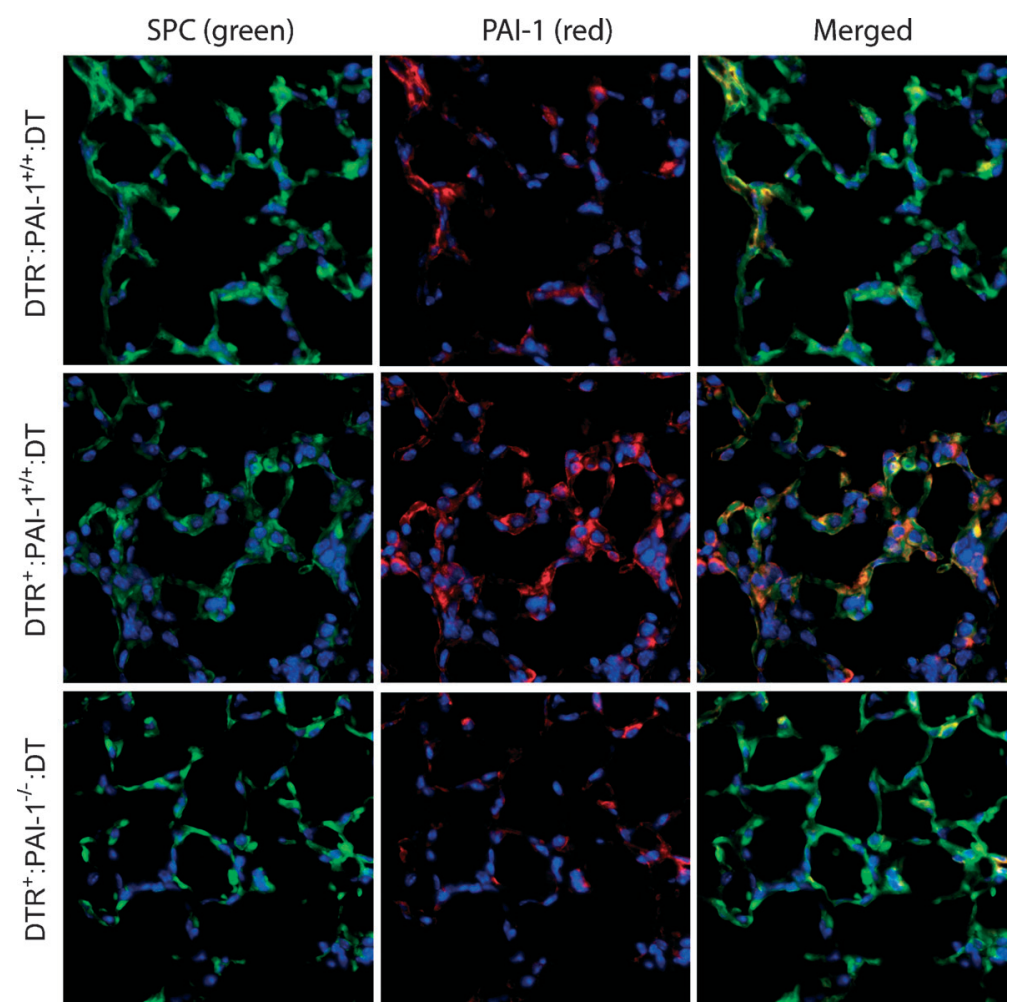

Figure 4. PAI-1 staining localizes to SPC-positive and SPC-negative cells following type II cell injury. Diphtheria toxin $(10.0 \mu \mathrm{g} / \mathrm{kg})$ was administered for 14 days/protocol to three strains of mice: $1, \mathrm{DTR}^{-}:$PAl-1 ${ }^{+/+} ; 2, \mathrm{DTR}^{+}$: PAl-1+/+ ; and $\mathrm{DTR}^{+}: \mathrm{PAI}-1^{-/-}$. On day 21 , lungs were harvested and $5 \mu \mathrm{m}$ sections were generated for PAI-1 and SPC immunofluorescence $(n=2-3)$. The primary antibodies to SPC were tagged with a green fluorescent protein and the primary antibody to PAI-1 was tagged with a red fluorescent antibody. Representative images from an individual mouse in each group are shown.

were then harvested for RNA and PAI-1 message level was measured using qualitative RT-PCR. We then compared the expression of treated versus untreated cells from each group. As demonstrated in Figure 5A, DT-injured DTR $^{+}$cells had a significant increase in PAI-1 message compared to unexposed controls. In contrast, non-transgenic cells showed a decrease in PAI-1 expression following DT treatment, and the difference in response between the two groups was highly statistically significant.

With the second approach, we isolated type II alveolar epithelial cells from $\mathrm{DTR}^{+}$and $\mathrm{DTR}^{-}$mice following 14 days of diphtheria toxin treatment. Type II cells were also collected from a control group of $\mathrm{DTR}^{-}$mice that received 14 days of i.p. saline. The cells from each group of mice were then cultured for 3 days prior to harvesting RNA for the quantification of PAI-1 message levels. The relative PAI-1 expression of our two DT treatment groups was compared to the uninjured control cells. We again found that mean PAI-1 mRNA expression was increased in the injured $\mathrm{DTR}^{+}$AECs as compared to the $\mathrm{DTR}^{-}$cells, although in this set of experiments the difference did not reach statistical significance (Figure 5B).

\section{PAI-1 expression by lung macrophages}

Our data indicate that type II AECs are a source of PAI-1 in response to DT-mediated injury of the alveolar epithelium. However, PAI-1 immunostaining also suggested an additional cellular source of expression in these mice. Many of these PAI-1positive: SPC-negative cells appeared large, and their microanatomical location suggested a macrophage origin (Figure 4, middle panels). To determine whether lung macrophages expressed PAI-1, we isolated two subpopulations of cells from $\mathrm{DTR}^{-}$and $\mathrm{DTR}^{+}$mice on day 14 of DT treatment (ie the time-point of maximal PAI-1 expression). These subsets included alveolar macrophages (large autofluorescent cells expressing CD11c but not CD11b) and exudate macrophages (large autofluorescent cells expressing both CD11c and CD11b) [38,40,41]. Both isolated subsets consisted of large cells with abundant cytoplasm and occasional intracellular vacuoles (Figure 6A, B). Expression of PAI-1 mRNA was detectable using qRT-PCR in both populations but appeared consistently higher in the exudate macrophages (Figure 6C). PAI-1 mRNA expression was comparable within each macrophage subset obtained from the $\mathrm{DTR}^{+}$and $\mathrm{DTR}^{-}$groups.

\section{Exudate macrophage accumulation}

Because the relative expression of PAI-1 was similar in the exudate macrophages from the $\mathrm{DTR}^{-}$and $\mathrm{DTR}^{+}$mice, we hypothesized that, if these cells were contributing to the increased BAL PAI-1 levels in the $\mathrm{DTR}^{+}$group, it may have been due to their 

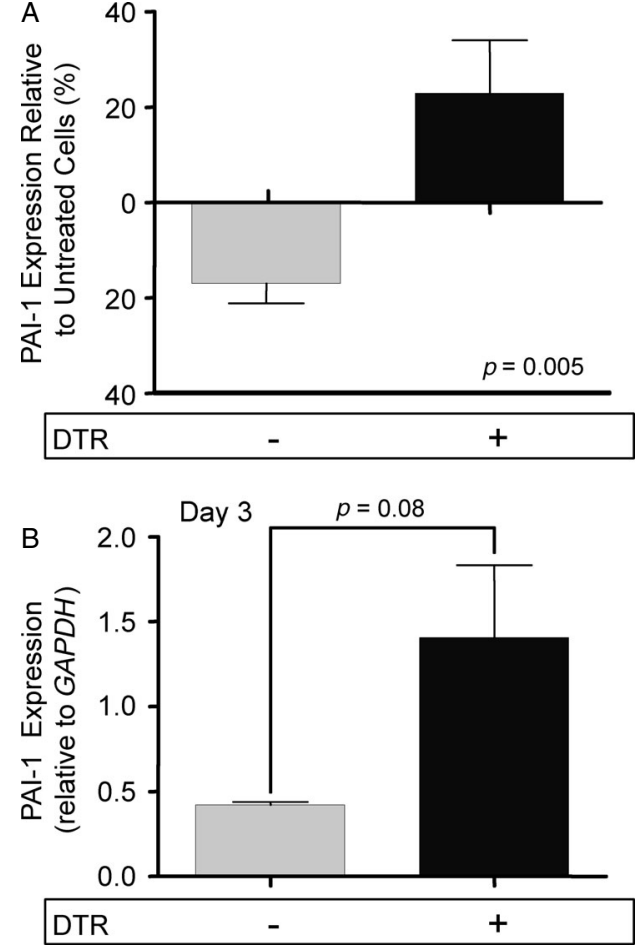

Figure 5. Epithelial cells express PAI-1 following DT-mediated injury. (A) Type II alveolar epithelial cells were isolated from $\mathrm{DTR}^{-}$and DTR+ mice. $5.0 \times 10^{5}$ cells were plated in wells precoated with fibronectin. After $48 \mathrm{~h}$, a subset of cells from each genotype was exposed to DT $(1.0 \mu \mathrm{g} / \mathrm{ml})$ for $24 \mathrm{~h}$. RNA was harvested from the cells and PAI- 1 expression was assessed via qRT-PCR and normalized to GAPDH. PAl-1 message levels from the treated cells were compared to untreated cells of the same genotype. Data are given as mean relative expression \pm SEM, $n=10 /$ group. (B) Diphtheria toxin $(10.0 \mu \mathrm{g} / \mathrm{kg})$ was administered for 14 days/protocol to $\mathrm{DTR}^{-}(n=3)$ and $\mathrm{DTR}^{+}$mice $(n=3)$. A control group of DTR- mice was treated with PBS for 14 days. Type II AECs were isolated from each group and $2.0 \times 10^{6}$ cells were cultured in fibronectin-coated wells. After $48 \mathrm{~h}$, RNA was harvested from the cells and PAI- 1 expression was assessed via qRT-PCR and normalized to GAPDH. PAI-1 message levels from the DT-treated groups were compared to the control. Data are given as mean relative expression $\pm \mathrm{SEM}, n=3 /$ group.

increased lung accumulation. To test this hypothesis, we quantified the number of exudate as well as alveolar macrophages within lung leukocyte populations from DT-treated $\mathrm{DTR}^{-}:$PAI- $1^{+/+}, \mathrm{DTR}^{+}:$PAI- $1^{+/+}$and $\mathrm{DTR}^{+}:$PAI-1 ${ }^{-/-}$mice on day 14 . We found that the alveolar macrophage population was not different between the three groups (Figure 7A). In contrast, exudate macrophage numbers increased significantly in the lungs of the DT-treated DTR ${ }^{+}:$PAI- $1^{+/+}$mice compared to the control group (Figure 7B). In the absence of PAI-1, however, exudate macrophages did not accumulate in DTR-expressing mice following DT injury (Figure 7B).

Exudate macrophages are derived from $\mathrm{Ly} 6 \mathrm{C}^{\text {high }}$ monocyte precursors $[38,40]$. To determine whether PAI-1 deficiency impacted exudate macrophage accumulation by regulating the recruitment versus the differentiation of Ly6C high monocytes, we next quantified these cells in the lung on day 14 of DT
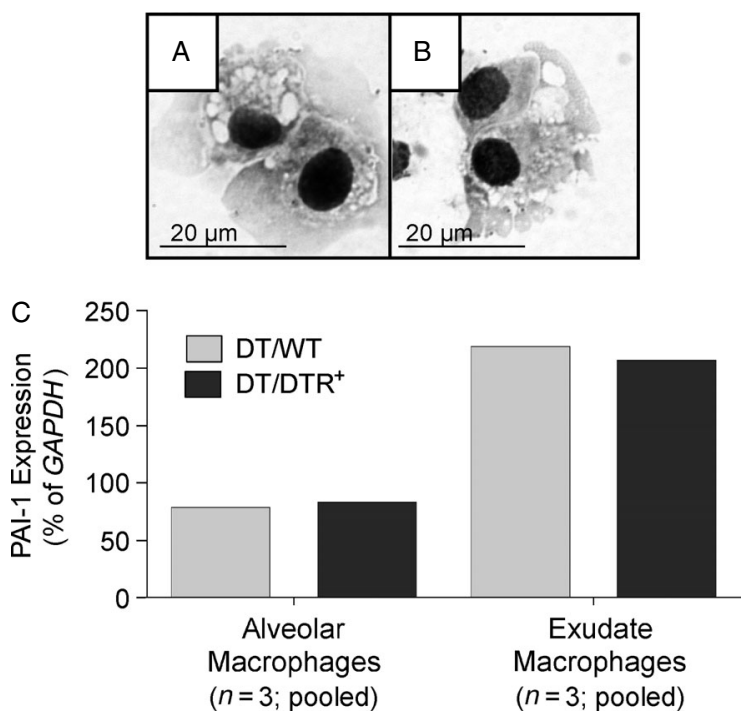

Figure 6. Alveolar and exudate lung macrophages express PAI1. $(A-C)$ Diphtheria toxin $(10.0 \mu \mathrm{g} / \mathrm{kg})$ was administered for 14 days/protocol to $\mathrm{DTR}^{-}(n=3)$ and $\mathrm{DTR}^{+}$mice $(n=3)$. Lung leukocytes from each strain of mice were isolated, pooled and antibody stained as described in Materials and methods. Multiparameter fluorescence-activated cell sorting was performed to eliminate non-macrophage populations and to further isolate two populations of autofluorescent lung macrophages: $C_{1} 11 \mathrm{c}^{+}$ $\mathrm{CD}_{11 \mathrm{~b}^{-}}$alveolar macrophages and $\mathrm{CD}_{11 \mathrm{c}^{+}} \mathrm{CD} 11 \mathrm{~b}^{+}$exudate macrophages. ( $A, B)$ Photomicrographs (taken with a $\times 1000$ objective) of sorted alveolar macrophages (A) and exudate macrophages (B). RNA was harvested from each macrophage subset and PAI-1 mRNA expression was assessed via qRT-PCR and normalized to GAPDH (C). Light grey bars, WT/DT; medium grey bars, DT/DTR ${ }^{+}$.

treatment. As demonstrated in Figure 7C, the number of Ly6 $\mathrm{C}^{\text {high }}$ monocytes was significantly increased in the $\mathrm{DTR}^{+}$mice as compared to WT mice following alveolar injury. In contrast, the accrual of this precursor monocyte population in PAI-1-deficient DTR-expressing mice was significantly impeded. This observation supports the conclusion that PAI-1 enhances the accumulation of exudate macrophages following type II AEC injury by facilitating Ly $6 \mathrm{C}^{\text {high }}$ monocyte recruitment.

\section{Collagen production by exudate macrophages}

We next assessed whether the lung macrophage populations of interest directly expressed collagen. First, we performed qRT-PCR to quantify type-1 collagen expression by alveolar and exudate macrophages isolated from the lungs of DT-injured $\mathrm{DTR}^{-}$and $\mathrm{DTR}^{+}$mice. The results showed that both alveolar macrophages and exudate macrophages express collagen-1 mRNA with higher levels observed in the exudate subset (Figure 8A).

We next used intracellular staining for collagen-1 to determine whether gene expression correlated with the presence of protein in the DT-treated $\mathrm{DTR}^{-}$: PAI$1^{+/+}, \mathrm{DTR}^{+}: \mathrm{PAI}-1^{+/+}$and $\mathrm{DTR}^{+}:$PAI- $1^{-/-}$groups on day 14. Although we observed that a substantial percentage (16-19\%) of alveolar macrophages stained for intracellular collagen $\left(\mathrm{Col1}^{+}\right.$; Figure 8B), neither 

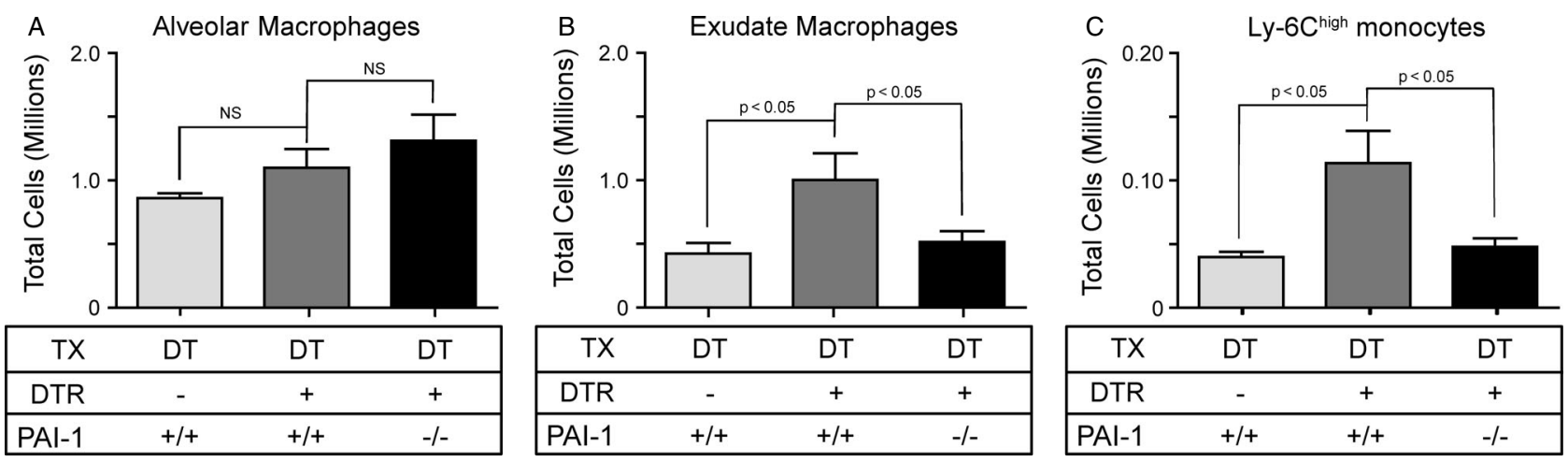

Figure 7. PAl-1 mediates the accumulation of exudate macrophages and Ly-6Chigh monocytes following alveolar injury. Diphtheria toxin $(10.0 \mu \mathrm{g} / \mathrm{kg})$ was administered for 14 days $/$ protocol to three strains of mice: $1, \mathrm{DTR}^{-}:$PAl- $1^{+/+} ; 2, \mathrm{DTR}^{+}: \mathrm{PAl}^{-1^{+/}} ;$and DTR $^{+}:$PAl- $1^{-/-}$. Lung leukocytes from individual mice were isolated and antibody stained. Multi-parameter flow cytometric analysis was used to identify and enumerate total numbers of alveolar macrophages (A), exudate macrophages (B) and Ly-6C ${ }^{\text {high }}$ monocytes (C). Data are given as mean \pm SEM of nine DT-treated mice/strain (from two separate experiments), assayed individually; light grey bars, DT/WT; medium grey bars, $\mathrm{DT}_{\mathrm{DTR}}{ }^{+}$; and dark grey bars, $\mathrm{DT} / \mathrm{DTR}^{+} / \mathrm{PAl}-1^{-}$. Values of $p<0.05$ compared by unpaired Student's $t$-test between groups at the same time point were considered significant.
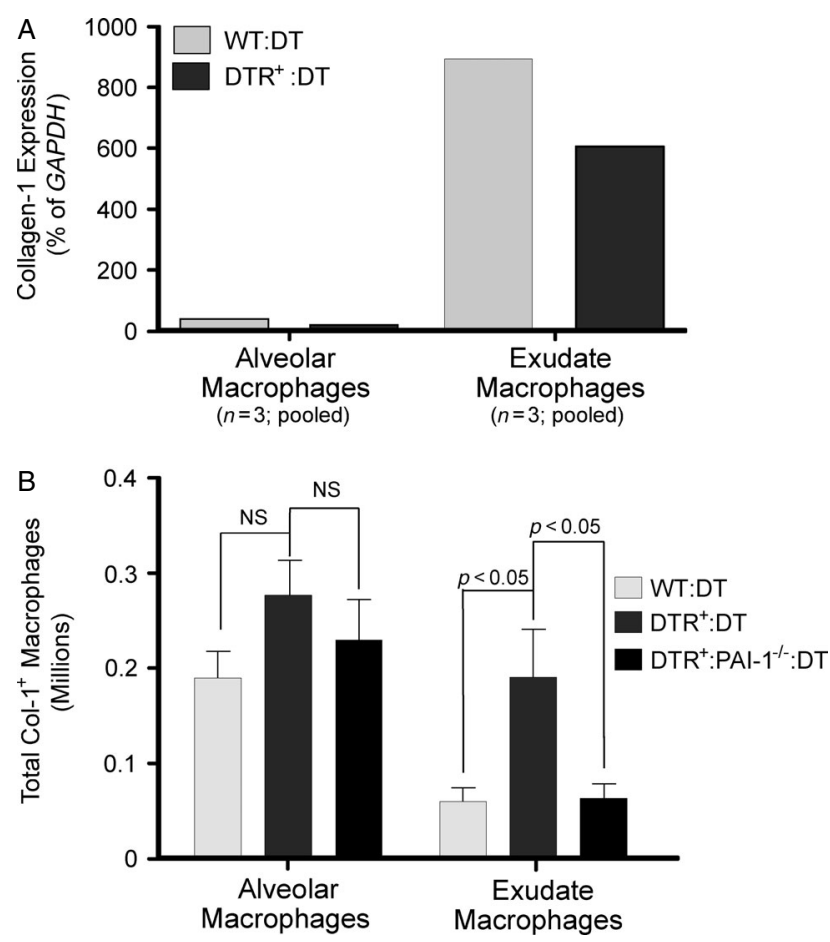

Figure 8. PAl-1 mediates the specific accumulation of collagen1-positive exudate macrophages in mice with DT-mediated alveoli. (A) Specific subsets of either alveolar macrophages or exudate macrophages were isolated (by cell sorting, as described in Materials and methods and Figure 8) from the lungs of DTtreated DTR- (light grey bars) and DTR- (medium grey bars) mice, and expression of Collagen-1 mRNA was assessed by qRT-PCR (normalized to GAPDH). (B) Diphtheria toxin $(10.0 \mu \mathrm{g} / \mathrm{kg})$ was administered for 14 days/protocol to three strains of mice: 1 , $\mathrm{DTR}^{-}: \mathrm{PAl}^{-1+/+} ; 2 \mathrm{DTR}^{+}: \mathrm{PAl}-1^{+/+}$; and $3 \mathrm{DTR}^{+}: \mathrm{PAl}-1^{-/-}$. Lung leukocytes from individual mice were isolated and antibody stained, using a protocol identifying intracellular collagen-1 protein. Multi-parameter flow cytometric analysis was used to identify and enumerate total numbers of collagen-1-positive alveolar macrophages or exudate macrophages. Data are given as mean \pm SEM of nine DT-treated mice/strain, from two separate experiments, assayed individually. Light grey bars, DT/DTR ${ }^{-}$; medium grey bars, $\mathrm{DT} \mathrm{DTR}^{+}$; and dark grey bars, DT/DTR ${ }^{+} / \mathrm{PAl}^{-} 1^{-}$). Values of $p<0.05$ compared by unpaired Student's $t$-test between groups at the same time point were considered significant. the percentage nor the total number of $\mathrm{Col1}^{+}$alveolar macrophages were increased in the DT-treated $\mathrm{DTR}^{+}: \mathrm{PAI}^{+/+}$mice relative to the other groups. In contrast, a higher percentage of exudate macrophages stained for intracellular collagen (relative to alveolar macrophages), with a trend towards the highest percentage of $\mathrm{Coll}^{+}$staining being observed in exudate macrophages obtained from DT-treated $\mathrm{DTR}^{+}$: PAI- $1^{+/+}$mice $(33 \pm 4 \%)$ [as compared to DT-treated controls $(27 \pm 4 \% ; p=0.28)$ and $\mathrm{DTR}^{+}: \mathrm{PAI}-1^{-/-}$ mice $(25 \pm 2 \% ; p=0.09)]$. Lastly, we calculated the total numbers of each $\mathrm{Col1}^{+}$macrophage subset. We found no difference in the number of $\mathrm{Col1}^{+}$alveolar macrophages between the three groups. In contrast, the total number of $\mathrm{Coll}^{+}$exudate macrophages was significantly higher in the DT-treated $\mathrm{DTR}^{+}$: PAI$1^{+/+}$mice relative to DT-treated control and $\mathrm{DTR}^{+}$: PAI- $1^{-/-}$groups.

\section{Discussion}

The role of PAI-1 in the pathogenesis of lung fibrosis was investigated using our model of fibrosis, initiated by targeted type II alveolar epithelial cell injury. We showed that an insult to the type II epithelium significantly induces PAI-1 expression in the lung. We also demonstrated that mice deficient in PAI-1 are markedly protected from epithelial injury-induced lung collagen accumulation and mortality. Furthermore, we determined that the cellular source of PAI-1 production in response to injury includes the type II AECs and two populations of lung macrophages - alveolar and exudate macrophages. In addition, we report the novel findings that PAI-1 itself mediates the accumulation of exudate macrophages and that these cells produce type I collagen. Lastly, our data revealed a strong association between the accumulation of the exudate macrophages and the development of pulmonary fibrosis in response to alveolar injury. 
Prior to the initiation of these experiments, the direct relationship between PAI-1 levels and the severity of pulmonary fibrosis had been established entirely in the bleomycin model. Therefore, the possibility remained that the causal link was dependent on features unique to bleomycin injury. As such, a primary goal of this study was to determine whether PAI-1 influenced the development of pulmonary fibrosis in a separate and mechanistically distinct murine model. To accomplish this goal, we employed our recently described type II AEC injury model. With this model, we found that DT-mediated type II epithelial cell damage leads to an increase in the quantity of PAI-1 within bronchoalveolar lavage fluid. This induction of PAI-1 expression mimics the elevated levels of intrapulmonary PAI-1 observed in patients with pulmonary fibrosis of varying aetiologies [14-16,18]. PAI-1 production is also increased in the lung following bleomycin-mediated injury [19,20,22-26,42]. To determine whether the enhanced PAI-1 production following type II AEC injury was required for fibrogenesis, we generated transgenic mice that expressed human DTR on their type II AECs and were deficient in PAI-1. We found that PAI-1 deficiency conferred significant protection in these mice against DT-mediated pulmonary fibrosis. In fact, the lung hydroxyproline and histology in the $\mathrm{DTR}^{+}$: PAI-1 $1^{-/-}$mice was not different from the control $\mathrm{DTR}^{-}$: $\mathrm{PAI}-1^{-/-}$group. Importantly, PAI-1 deficiency also limited the morbidity/mortality that results from the targeted type II cell insult. This decreased morbidity/mortality in the DT-treated $\mathrm{DTR}^{+}$: PAI- $1^{-/-}$mice is reminiscent of that observed in PAI-1-deficient mice following both bleomycin- and hyperoxia-induced lung injury [25,43]. Of note, PAI-1 inhibition was very recently reported to reduce fibrosis in yet another model of pulmonary fibrosis, intrapulmonary TGF $\beta$ over-expression [28]. Together, these observations provide very strong evidence substantiating a critical and fundamental role for PAI-1 in the pathogenesis of pulmonary fibrosis.

Aspects of our animal model are distinct from bleomycin-mediated injury and share features of pathogenesis with the human disease which contribute to the significance of our findings. Most importantly, the DTR model recapitulates the epithelial injury that is such a prominent histopathological feature of the human disease [30-32]. In fact, the generation of our DTR model was motivated by a popular hypothesis that type II AEC defects are critical for the development of lung fibrosis [32]. This hypothesis is supported by the consistently recognized abnormalities (eg denudation and hypertrophy) in the alveolar epithelium overlying fibroblast foci, the purported lesion of active scarring. The association between mutations in type II alveolar epithelial cell-specific genes and the development of familial IPF lends additional credence to this hypothesis $[33,34]$. Because the type II AEC injury model shares pathogenic features with IPF, we believe our data enhance the relevance of PAI-1 as a potential therapeutic target.
Our data revealed that at least two cellular sources, type II AEC and lung macrophages, contribute to the increased PAI-1 levels in the alveolar compartment of DT-injured $\mathrm{DTR}^{+}$mice. As with our original report, we again observed persistent SPC-expressing cells in the lungs of $\mathrm{DTR}^{+}$mice following DT administration. Importantly, these residual type II AECs contribute to the pro-fibrotic milieu through their production of PAI-1. Consistent with published literature, our present data indicate that these cells are not the only source of PAI-1 in the injured lung. In addition to type II AECs, we found that macrophages also produce PAI-1. This latter observation is consistent with data from the bleomycin model, in which microdissected type II AECs expressed 10-fold higher levels of PAI-1, while macrophages obtained either by lavage or by microdissection expressed $\sim 20-60$-fold higher PAI-1 levels [44]. In the present study, we further defined the production of PAI- 1 by macrophage subsets and found that the accumulated non-resident exudate population most strongly expressed PAI-1. Although our results clearly implicate type II AECs and exudate macrophages as important sources of PAI-1 in the lung following a targeted epithelial injury, our results do not exclude the participation of other cells, such as fibroblasts, in the production of PAI-1.

Although many studies implicate PAI-1 as a potent fibrogeneic mediator, the elucidation of its mechanism of action in promoting collagen accumulation has been elusive. Herein, we provide results that further clarify the role PAI-1 in fibrogenesis. We report the novel finding that PAI-1 is necessary for the accumulation of exudate macrophages in the lung following type II AEC injury. These cells express collagen 1 mRNA and stain for intracellular collagen, suggesting that they contribute directly to the fibrotic process. Our data reveal that PAI-1 facilitates the accrual of exudate macrophages by mediating the recruitment of Ly6 $\mathrm{C}^{\text {high }}$ monocytes, the precursor of exudate macrophages, into the injured lung. There exist many plausible mechanisms by which PAI-1 might influence this monocyte recruitment. For example, PAI-1 may enhance the transendothelial migration of Ly6C $\mathrm{C}^{\text {high }}$ monocytes across the pulmonary vasculature (perhaps through its vitronectin-binding function) [42]. Alternatively, PAI-1 may alter the expression or recognition of monocyte/macrophage chemotactic factors (ie CCL2 or CCL7). A potential effect of PAI-1 on monocyte development within the bone marrow also warrants consideration. Future studies are planned to further delineate these potential mechanisms.

In summary, we have determined that a targeted type II cell injury leads to the significant induction of PAI-1 and that PAI-1 is necessary for the subsequent fibrotic response. These findings establish a causal role for PAI-1 in a model of pulmonary fibrosis other than bleomycin, and implicate PAI-1 as a central component of the fibrogenic pathway. Our data further implicate the PAI-1 mediated accumulation of exudate macrophages as one potential mechanism whereby 
PAI-1 contributes to the development of lung fibrosis. Ultimately these findings further motivate the targeting of PAI-1 as a therapeutic strategy for human fibrotic disorders.

\section{Acknowledgment}

We would like to acknowledge our funding sources, which include the National Institutes of Health (Grant No. 1 R01 HL078871, to THS), the Quest For Breath Foundation (to THS), Veterans Administration Career Development Award-2 (to JJO), National Institutes of Health (Grant No. P01 HL089407, to DAL, Grant No. K08 HL081059, to JCH and Grant No. R01HL105489, to JCH). We would also like to acknowledge Bi Yu for his work on preliminary studies.

\section{Author contributions}

JJO planned and performed experiments to quantify macrophage accumulation and to analyse macrophage expression of PAI-1 and collagen, and also assisted with manuscript preparation; PJC planned and performed experiments to analyse PAI-1 expression by injured type II alveolar epithelial cells; VL planned and performed experiments to co-immunostain PAI1 and surfactant protein $\mathrm{C}$; JCH assisted in experimental planning and data interpretation for the entire manuscript, and also assisted with manuscript preparation; $\mathrm{NH}$ assisted in experimental planning and data interpretation for the entire manuscript; NS performed all of the in vivo experiments; AC assisted with all of the in vivo experiments and managed the breeding colonies for the DTR mice; YL assisted with all of the in vivo experiments and managed the breeding colonies for the DTR mice; BJM assisted with experiments to quantify macrophage accumulation and to analyse macrophage expression of PAI- 1 and collagen; REM assisted with experiments to quantify macrophage accumulation and to analyse macrophage expression of PAI-1 and collagen; MAO assisted with experiments to quantify macrophage accumulation and to analyse macrophage expression of PAI-1 and collagen; DAL performed experiments to quantify PAI1 levels in DTR mice following targeted epithelial cell injury; RHS assisted in experimental planning and data interpretation for the entire manuscript, and also assisted with manuscript preparation; THS generated the hypothesis, planned the experiments, interpreted the data for the entire manuscript and was also the primary author.

\section{References}

1. Selman M, Pardo A, Richeldi L, et al. Emerging drugs for idiopathic pulmonary fibrosis. Expert Opin Emerg Drugs 2011; 16: 341-362.
2. Azuma A, Nukiwa T, Tsuboi E, et al. Double-blind, placebocontrolled trial of pirfenidone in patients with idiopathic pulmonary fibrosis. Am J Respir Crit Care Med 2005; 171: 1040-1047.

3. Noble PW, Albera C, Bradford WZ, et al. Pirfenidone in patients with idiopathic pulmonary fibrosis (CAPACITY): two randomised trials. Lancet 2011; 377: 1760-1769.

4. Richeldi L, Costabel U, Selman M, et al. Efficacy of a tyrosine kinase inhibitor in idiopathic pulmonary fibrosis. $N$ Engl $J$ Med 2011; 365: 1079-1087.

5. Ventilation with lower tidal volumes as compared with traditional tidal volumes for acute lung injury and the acute respiratory distress syndrome. The Acute Respiratory Distress Syndrome Network. $N$ Engl J Med 2000; 342: 1301-1308.

6. Brower RG, Lanken PN, MacIntyre N, et al. Higher versus lower positive end-expiratory pressures in patients with the acute respiratory distress syndrome. $N$ Engl J Med 2004; 351: 327-336.

7. Steinberg KP, Hudson LD, Goodman RB, et al. Efficacy and safety of corticosteroids for persistent acute respiratory distress syndrome. N Engl J Med 2006; 354: 1671-1684.

8. Wheeler AP, Bernard GR, Thompson BT, et al. Pulmonary artery versus central venous catheter to guide treatment of acute lung injury. N Engl J Med 2006; 354: 2213-2224.

9. Wiedemann HP, Wheeler AP, Bernard GR, et al. Comparison of two fluid-management strategies in acute lung injury. $N$ Engl $\mathrm{J} \mathrm{Med}$ 2006; 354: 2564-2575.

10. Flaherty KR, Thwaite EL, Kazerooni EA, et al. Radiological versus histological diagnosis in UIP and NSIP: survival implications. Thorax 2003; 58: 143-148.

11. Olson AL, Swigris JJ, Raghu G, et al. Seasonal variation: mortality from pulmonary fibrosis is greatest in the winter. Chest 2009; 136: $16-22$.

12. Frankel SK, Schwarz MI. Update in idiopathic pulmonary fibrosis. Curr Opin Pulm Med 2009; 15: 463-469.

13. Chapman HA, Allen CL, Stone OL. Abnormalities in pathways of alveolar fibrin turnover among patients with interstitial lung disease. Am Rev Respir Dis 1986; 133: 437-443.

14. Idell S, James KK, Levin EG, et al. Local abnormalities in coagulation and fibrinolytic pathways predispose to alveolar fibrin deposition in the adult respiratory distress syndrome. J Clin Invest 1989; 84: 695-705.

15. Bertozzi P, Astedt B, Zenzius L, et al. Depressed bronchoalveolar urokinase activity in patients with adult respiratory distress syndrome. N Engl J Med 1990; 322: 890-897.

16. Kotani I, Sato A, Hayakawa $\mathrm{H}$, et al. Increased procoagulant and antifibrinolytic activities in the lungs with idiopathic pulmonary fibrosis. Thromb Res 1995; 77: 493-504.

17. Sisson TH, Simon RH. The plasminogen activation system in lung disease. Curr Drug Targets 2007; 8: 1016-1029.

18. Prabhakaran P, Ware LB, White KE, et al. Elevated levels of plasminogen activator inhibitor-1 in pulmonary edema fluid are associated with mortality in acute lung injury. Am J Physiol Lung Cell Mol Physiol 2003; 285: L20-28.

19. Idell S, James KK, Gillies C, et al. Abnormalities of pathways of fibrin turnover in lung lavage of rats with oleic acid and bleomycininduced lung injury support alveolar fibrin deposition. Am J Pathol 1989; 137: 387-389.

20. Olman MA, Mackman N, Gladson CL, et al. Changes in procoagulant and fibrinolytic gene expression during bleomycin-induced lung injury in the mouse. J Clin Invest 1995; 96: 1621-1630.

21. Nishiuma T, Sisson TH, Subbotina N, et al. Localization of plasminogen activator activity within normal and injured lungs by in situ zymography. Am J Respir Cell Mol Biol 2004; 31: 552-558.

22. Eitzman DT, McCoy RD, Zheng X, et al. Bleomycin-induced pulmonary fibrosis in transgenic mice that either lack or overexpress 
the murine plasminogen activator inhibitor-1 gene. J Clin Invest 1996; 97: 232-237.

23. Sisson TH, Hattori N, Xu Y, et al. Treatment of bleomycininduced pulmonary fibrosis by transfer of urokinase-type plasminogen activator genes. Hum Gene Ther 1999; 10: 2315-2323.

24. Sisson TH, Hanson KE, Subbotina N, et al. Inducible lungspecific urokinase expression reduces fibrosis and mortality after lung injury in mice. Am J Physiol Lung Cell Mol Physiol 2002; 283: L1023-1032.

25. Hattori N, Degen JL, Sisson TH, et al. Bleomycin-induced pulmonary fibrosis in fibrinogen-null mice. J Clin Invest 2000; 106: $1341-1350$

26. Gunther A, Lubke N, Ermert M, et al. Prevention of bleomycininduced lung fibrosis by aerosolization of heparin or urokinase in rabbits. Am J Respir Crit Care Med 2003; 168: 1358-1365.

27. Senoo T, Hattori N, Tanimoto T, et al. Suppression of plasminogen activator inhibitor- 1 by RNA interference attenuates pulmonary fibrosis. Thorax 2010; 65: 334-340.

28. Huang WT, Vayalil PK, Miyata T, et al. Therapeutic value of small molecule inhibitor to plasminogen activator inhibitor-1 for lung fibrosis. Am J Respir Cell Mol Biol 2012; 46: 87-95.

29. Sisson TH, Mendez M, Choi K, et al. Targeted injury of type II alveolar epithelial cells induces pulmonary fibrosis. Am J Respir Crit Care Med 2010; 181: 254-263.

30. Katzenstein AA. Pathogenesis of 'fibrosis' in interstitial pneumonia: an electron microscopic study. Human Pathol 1985; 16: 1015-1024.

31. Kasper M, Haroske G. Alterations in the alveolar epithelium after injury leading to pulmonary fibrosis. Histol Histopathol 1996; 11: 463-483.

32. Selman M, Pardo A. Role of epithelial cells in idiopathic pulmonary fibrosis: from innocent targets to serial killers. Proc Am Thorac Soc 2006; 3: 364-372.

33. Thomas AQ, Lane K, Phillips J, 3rd, et al. Heterozygosity for a surfactant protein $\mathrm{C}$ gene mutation associated with usual interstitial pneumonitis and cellular nonspecific interstitial pneumonitis in one kindred. Am J Respir Crit Care Med 2002; 165: $1322-1328$.
34. Maitra M, Wang Y, Gerard RD, et al. Surfactant protein A2 mutations associated with pulmonary fibrosis lead to protein instability and endoplasmic reticulum stress. J Biol Chem 2010; 285: 22103-22113

35. Carmeliet P, Kieckens L, Schoonjans L, et al. Plasminogen activator inhibitor-1 gene-deficient mice. I. Generation by homologous recombination and characterization. J Clin Invest 1993; 92: 2746-2755

36. Lazar MH, Christensen PJ, Du M, et al. Plasminogen activator inhibitor-1 impairs alveolar epithelial repair by binding to vitronectin. Am J Respir Cell Mol Biol 2004; 31: 672-678.

37. Osterholzer JJ, Curtis JL, Polak T, et al. CCR2 mediates conventional dendritic cell recruitment and the formation of bronchovascular mononuclear cell infiltrates in the lungs of mice infected with Cryptococcus neoformans. J Immunol 2008; 181: 610-620.

38. Osterholzer JJ, Chen GH, Olszewski MA, et al. Chemokine receptor 2-mediated accumulation of fungicidal exudate macrophages in mice that clear cryptococcal lung infection. Am J Pathol 2011; 178: $198-211$

39. Moore BB, Murray L, Das A, et al. The role of CCL12 in the recruitment of fibrocytes and lung fibrosis. Am J Respir Cell Mol Biol 2006; 35: 175-181.

40. Lin KL, Suzuki Y, Nakano H, et al. CCR2+ monocyte-derived dendritic cells and exudate macrophages produce influenza-induced pulmonary immune pathology and mortality. J Immunol 2008; 180: $2562-2572$

41. Tighe RM, Liang J, Liu N, et al. Recruited exudative macrophages selectively produce CXCL10 after noninfectious lung injury. Am J Respir Cell Mol Biol 2011; 45: 781-788.

42. Courey AJ, Horowitz JC, Kim KK, et al. The vitronectin-binding function of PAI-1 exacerbates lung fibrosis in mice. Blood 2011, 118: $2313-2321$.

43. Barazzone C, Belin D, Piguet PF, et al. Plasminogen activator inhibitor-1 in acute hyperoxic mouse lung injury. $J$ Clin Invest 1996; 98: 2666-2673.

44. Wygrecka M, Markart P, Ruppert C, et al. Cellular origin of procoagulant and (anti)-fibrinolytic factors in bleomycin-injured lungs. Eur Respir J 2007; 29: 1105-1114.

\section{SUPPORTING INFORMATION ON THE INTERNET}

The following supporting information may be found in the online version of this article:

\section{Supplementary materials and methods}

Figure S1. PAI-1 is associated with decreased survival following type II AEC injury. 